\title{
LIPSCHITZ HOMOTOPY AND DENSITY OF LIPSCHITZ MAPPINGS IN SOBOLEV SPACES
}

\author{
Piotr Hajłasz and Armin Schikorra
}

\author{
University of Pittsburgh, Department of Mathematics \\ 301 Thackeray Hall, Pittsburgh, PA 15260, U.S.A.; hajlasz@pitt.edu \\ Max-Planck Institut MiS Leipzig \\ Inselstr. 22, 04103 Leipzig, Germany; armin.schikorra@mis.mpg.de
}

\begin{abstract}
We construct a smooth compact $n$-dimensional manifold $Y$ with one point singularity such that all its Lipschitz homotopy groups are trivial, but Lipschitz mappings $\operatorname{Lip}\left(\mathbf{S}^{n}, Y\right)$ are not dense in the Sobolev space $W^{1, n}\left(\mathbf{S}^{n}, Y\right)$. On the other hand we show that if a metric space $Y$ is Lipschitz $(n-1)$-connected, then Lipschitz mappings $\operatorname{Lip}(X, Y)$ are dense in $N^{1, p}(X, Y)$ whenever the Nagata dimension of $X$ is bounded by $n$ and the space $X$ supports the $p$-Poincaré inequality. Lipschitz $(n-1)$-connectedness is a stronger condition than vanishing of the first $n-1$ Lipschitz homotopy groups as it assumes quantitative estimates of Lipschitz constants.
\end{abstract}

\section{Introduction}

Let $\mathcal{M}$ and $\mathcal{N}$ be compact Riemannian manifolds, $\partial \mathcal{N}=\emptyset$. Being motivated by the theory of harmonic mappings Eells and Lemaire, [8], asked a question whether smooth mappings $C^{\infty}(\mathcal{M}, \mathcal{N})$ are dense in the space of Sobolev mappings between manifolds $W^{1, p}(\mathcal{M}, \mathcal{N})$. Here we assume that $\mathcal{N}$ is isometrically embedded in an Euclidean space $\mathbf{R}^{\nu}$ and we define

$$
W^{1, p}(\mathcal{M}, \mathcal{N})=\left\{u \in W^{1, p}\left(\mathcal{M}, \mathbf{R}^{\nu}\right): u(x) \in \mathcal{N} \text { a.e. }\right\} .
$$

The space is equipped with the metric of $W^{1, p}\left(\mathcal{M}, \mathbf{R}^{\nu}\right)$. Equivalently one may ask about density of Lipschitz mappings. This is indeed an equivalent question, because Lipschitz mappings can be approximated by smooth mappings in the Sobolev norm. If $p \geq \operatorname{dim} \mathcal{M}$, then smooth mappings are dense in $W^{1, p}(\mathcal{M}, \mathcal{N})$, by the theorem of Schoen and Uhlenbeck [33, 34], but if $p<\operatorname{dim} \mathcal{M}$, the answer depends on the topology of manifolds $\mathcal{M}$ and $\mathcal{N}$. The following necessary condition for the density is due to Bethuel and Zheng [3].

Proposition 1.1. If $\pi_{[p]}(\mathcal{N}) \neq\{0\}$ and $1 \leq p<\operatorname{dim} \mathcal{M}$, then the smooth mappings $C^{\infty}(\mathcal{M}, \mathcal{N})$ are not dense in $W^{1, p}(\mathcal{M}, \mathcal{N})$.

Here $\pi_{k}$ stands for the homotopy group and $[p]$ is the integral part of $p$. This result is relatively easy to prove (see also [14,11]). Bethuel [2] proved that in the local case (mappings from a ball) this condition is also sufficient. The proof of sufficiency is, however, very difficult (see [19] for corrections to Bethuel's paper).

Theorem 1.2. If $1 \leq p<n$, then smooth mappings $C^{\infty}\left(\mathbf{B}^{n}, \mathcal{N}\right)$ are dense in $W^{1, p}\left(\mathbf{B}^{n}, \mathcal{N}\right)$ if and only if $\pi_{[p]}(\mathcal{N})=0$.

doi:10.5186/aasfm.2014.3932

2010 Mathematics Subject Classification: Primary 46E35; Secondary 55Q70.

Key words: Sobolev mappings, density, Lipschitz homotopy groups, metric spaces.

P.H. was supported by NSF grant DMS-1161425. A.S. was supported by DAAD fellowship $\mathrm{D} / 12 / 40670$. 
In the general case of $W^{1, p}(\mathcal{M}, \mathcal{N}), 1 \leq p<\operatorname{dim} \mathcal{M}$, the necessary condition $\pi_{[p]}(\mathcal{N})=0$ is not always sufficient for the density, see [18] for an example. The necessary and sufficient condition has been discovered by Hang and Lin in [19]. While we will not state this condition here, we will state a sufficient condition that was obtained earlier in [14], because this condition will play a role in what is to follow.

Proposition 1.3. If $\pi_{1}(\mathcal{N})=\pi_{2}(\mathcal{N})=\ldots=\pi_{[p]}(\mathcal{N})=0$, then $C^{\infty}(\mathcal{M}, \mathcal{N})$ mappings are dense in $W^{1, p}(\mathcal{M}, \mathcal{N})$.

If $p \geq \operatorname{dim} \mathcal{M}$, density is always true, so the interesting case is when $1 \leq p<$ $\operatorname{dim} \mathcal{M}$.

The theory of Sobolev mappings between manifolds has been extended to the case of Sobolev mappings with values into metric spaces. The first papers on this subject include the work of Ambrosio, [1], on limits of classical variational problems and the work of Gromov and Schoen, [9], on Sobolev mappings into the BruhatTits buildings, with applications to rigidity questions for discrete groups. Later the theory of Sobolev mappings with values into metric spaces was developed in a more elaborated form by Korevaar and Schoen, [29], in their approach to the theory of harmonic mappings into Alexandrov spaces of non-positive curvature. Other papers on Sobolev mappings from a manifold into a metric space include [5, 7, 24, 25, 26, 27, 32, 35]. Finally, analysis on metric spaces, the theory of Carnot-Carathéodory spaces and the theory of quasiconformal mappings between metric spaces led to the theory of Sobolev mappings between metric spaces, [22, 23, 30, 37], among which the theory of Newtonian-Sobolev mappings $N^{1, p}(X, Y)$ is particularly important.

The question in what way the results about density of smooth mappings between manifolds can be generalized to the case of mappings between metric spaces was formulated by Heinonen, Koskela, Shanmugalingam and Tyson, [23]. In this context one asks about density of Lipschitz mappings, because the class of smooth mappings make no sense. In order to formulate the problem we need to define the class $N^{1, p}(X, Y)$ of Sobolev mappings between metric spaces.

A metric-measure space $(X, d, \mu)$ is a metric space $(X, d)$ equipped with a Borelregular measure $\mu$. We say that the measure $\mu$ is doubling if there is a constant $C_{d} \geq 1$ such that for every ball $B$ in $X, \mu(2 B) \leq C_{d} \mu(B)$. Here and in what follows by $\sigma B, \sigma>0$ we denote the ball concentric with $B$ and with the radius $\sigma$ times that of $B$. In the paper we will always assume that the measure $\mu$ is doubling. For simplicity we will also always assume that the diameter of $X$ is finite. Note that this and the doubling condition imply $\mu(X)<\infty$.

Let $U \subset X$ be open. Following [22] we say that a Borel function $g: U \rightarrow[0, \infty]$ is an upper gradient of a Borel function $u$ defined in $U$ if

$$
|u(\gamma(b))-u(\gamma(a))| \leq \int_{\gamma} g
$$

for all rectifiable curves $\gamma:[a, b] \rightarrow U$. Then we say that the space $(X, d, \mu)$ supports the p-Poincaré inequality, $1 \leq p<\infty$ if there are constants $C_{P}>0$ and $\sigma \geq 1$ such that for every ball $B$ in $X$, every $u \in L^{1}(\sigma B)$ and every upper gradient $g$ of $u$ in $\sigma B$ the following version of the Poincaré inequality is satisfied

$$
f_{B}\left|u-u_{B}\right| d \mu \leq C_{P}(\operatorname{diam} B)\left(f_{\sigma B} g^{p} d \mu\right)^{1 / p} .
$$


Here the barred integral denotes the integral average and $u_{B}$ is the integral average of $u$ over $B$.

The Sobolev space $N^{1, p}(X, d, \mu)$ was introduced in [36] and it is defined as follows. We say that $u \in N^{1, p}(X, d, \mu)$ if $u \in L^{p}$ and there is an upper gradient $g \in L^{p}$ of $u$. The space is equipped with a norm which turns the space into a Banach space:

$$
\|u\|_{N^{1, p}}=\|u\|_{p}+\inf _{g}\|g\|_{p} .
$$

Here the infimum is taken over all upper gradients of $u$. To be more precise we have to identify functions $u, v \in N^{1, p}$ such that $\|u-v\|_{N^{1, p}}=0$, just like we identify functions in $L^{p}$ that are equal almost everywhere.

If $(V,\|\cdot\|)$ is a Banach space, then the vector valued Sobolev space $N^{1, p}(X, V)$ is defined in a similar manner [23]: $u \in N^{1, p}(X, V)$ if $u \in L^{p}(X, V)$ and there is $0 \leq g \in L^{p}(X)$ such that

$$
\|u(\gamma(b))-u(\gamma(a))\| \leq \int_{\gamma} g
$$

for all rectifiable curves $\gamma:[a, b] \rightarrow X$. Then $N^{1, p}(X, V)$ is a Banach space with respect to the norm (1.1). Here we also call $g$ an upper gradient of $u$. For more details, see [23]. If $X=\mathcal{M}$ is a Riemannian manifold and $V$ is dual to a separable Banach space, then this definition is equivalent to the classical definition of the vector valued Sobolev space $W^{1, p}(\mathcal{M}, V)$ via the distributional derivatives, see $[6,17]$.

In the case of real valued functions the next result was proved in [15, Theorem 3.2] from the Poincaré inequality via the telescoping argument. It was extended to the Banach space valued case in [23, Proposition 4.6]. Recall that we assumed that $\operatorname{diam} X<\infty$.

Proposition 1.4. Suppose that the space $(X, d, \mu)$ supports the p-Poincare inequality, $(V,\|\cdot\|)$ is a Banach space, $u \in N^{1, p}(X, V)$, and $g$ is an upper gradient of $u$. Then the pointwise inequality

$$
\|u(x)-u(y)\| \leq C d(x, y)\left(\left(\mathbf{M} g^{p}(x)\right)^{1 / p}+\left(\mathbf{M} g^{p}(y)\right)^{1 / p}\right)
$$

holds a.e. with some constant $C$ independent of $u$ and $g$. Here $\mathbf{M}$ stands for the Hardy-Littlewood maximal operator which is defined by

$$
\mathbf{M} h(x)=\sup _{r>0} f_{B(x, r)}|h| d \mu .
$$

This inequality implies that on the set $\left\{\left(\mathbf{M} g^{p}\right)^{1 / p} \leq t\right\}$ the function $u$ is $2 C t$ Lipschitz continuous. Using the Whitney decomposition of the complement of the set and the associated Lipschitz partition of unity one can extend the function $u$ to a Lipschitz function from $X$ to $V$. Since the Lipschitz function differs from $u$ on a set of small measure standard estimates lead to the following known result (see e.g. [12, Lemma 13]).

Proposition 1.5. Suppose that the space $(X, d, \mu)$ supports the $p$-Poincare inequality for some $1 \leq p<\infty$ and $V$ is a Banach space. If $u \in N^{1, p}(X, V)$, then for every $\varepsilon>0$ there is $u_{\varepsilon} \in \operatorname{Lip}(X, V)$ such that $\mu\left(\left\{x: u(x) \neq u_{\varepsilon}(x)\right\}\right)<\varepsilon$ and $\left\|u-u_{\varepsilon}\right\|_{1, p}<\varepsilon$.

Every separable metric space admits an isometric embedding into a Banach space $V$ which is dual to a separable Banach space. For example it can always be embedded 
into the Banach space $\ell^{\infty}$ of bounded sequences (the Kuratowski embedding). This can be used to define the space of Sobolev mappings $N^{1, p}(X, Y)$ with values in $Y$. Namely if $\lambda: Y \rightarrow V$ is an isometric embedding then we define

$$
N^{1, p}(X, Y)=\left\{u: X \rightarrow Y: \lambda \circ u \in N^{1, p}(X, V)\right\} .
$$

The space is equipped with the metric $d(u, v)=\|\lambda \circ u-\lambda \circ v\|_{N^{1, p}}$. This definition resembles the definition of the class of Sobolev mappings between smooth manifolds, but now instead of taking an embedding of the manifold into a Euclidean space we take an embedding of a metric space into a Banach space. It is important to point out that the space $N^{1, p}(X, Y)$ as a class of mappings does not depend on the particular embedding of $Y$ into $V$, because it can be characterized intrinsically in a way that does not involve any embedding of $Y$, [23, Corollary 3.21]. However, the metric in $N^{1, p}(X, Y)$ does depend on the embedding, [10, 13].

If $X=\mathcal{M}$ is a Riemannian manifold and $Y$ is embedded in a Banach space $V$ that is dual to a separable Banach space, then we will write $W^{1, p}(\mathcal{M}, Y)$, because, as it was explained earlier, we have $N^{1, p}(\mathcal{M}, V)=W^{1, p}(\mathcal{M}, V)$.

Observe that each mapping $u \in N^{1, p}(X, Y)$ (or rather $\lambda \circ u$ ) can be approximated by Lipschitz mappings with values into $V$ (Proposition 1.5). In this setting Heinonen, Koskela, Shanmugalingam and Tyson [23, Remark 6.9] asked: It is an interesting problem to determine when one can choose the Lipschitz approximation to have values in the target $Y$. [...] For instance, one can ask to what extent Bethuel's results have analogs for general targets.

As we pointed out the metric in $N^{1, p}(X, Y)$ depends on the choice of an embedding of $Y$ into $V$. Actually an example constructed in [10] (see also [13]) shows that the answer to the density question may also depend on an embedding. However, there is a large class of spaces described in [12, Theorem 4] for which it does not matter what embedding we choose.

The following partial answer to the question of Heinonen, Koskela, Shanmugalingam and Tyson was obtained in [12, Theorem 6].

Theorem 1.6. Let $Y$ be a finite Lipschitz polyhedron and $1 \leq p<\infty$. Then the class of Lipschitz mappings $\operatorname{Lip}(X, Y)$ is dense in $N^{1, p}(X, Y)$ for every metricmeasure space $X$ of finite measure that supports the $p$-Poincare inequality if and only if $\pi_{1}(Y)=\pi_{2}(Y)=\ldots=\pi_{[p]}(Y)=0$.

This is an example where it does not matter what embedding of $Y$ we choose.

Note that the condition from the theorem appeared also in Proposition 1.3, but now it is necessary and sufficient. For example if $Y$ is a compact manifold, we still get a necessary and sufficient condition in Theorem 1.6, differently than in Proposition 1.3. The reason for this difference is that in the proof of necessity in Theorem 1.6 we assume density for all spaces $X$ that support the $p$-Poincaré inequality, but in Proposition 1.3 we would only have density for a given manifold $\mathcal{M}$ which is a much weaker condition.

While the theorem treats a general class of spaces $X$ as a source, the targets are Euclidean-like, and it is an interesting question to investigate more general targets. Of particular interest is the Heisenberg group $\mathbf{H}_{n}$ which is a fundamental example of a metric space in the analysis on metric spaces. A motivation for the investigation of density of Lipschitz mappings in $N^{1, p}\left(X, \mathbf{H}_{n}\right)$ stems from the theory of harmonic mappings with values in the Heisenberg group developed by Capogna and Lin [5], just 
like the theory of harmonic mappings was the original motivation for the questions of Eells and Lemaire. This problem was investigated in $[6,16]$.

In the case of mappings between manifolds, the answer to the density question depends on the topology of manifolds and in particular on the homotopy groups of the target, see Proposition 1.3 and Theorem 1.6. The Heisenberg group $\mathbf{H}_{n}$ is homeomorphic to the Euclidean space $\mathbf{R}^{2 n+1}$ and thus its homotopy groups are trivial. It turns out, however, that more appropriate objects to consider in the case of metric targets are the Lipschitz homotopy groups. For the following definition, see [6].

Definition 1.7. Let $\left(Y, y_{0}\right)$ be a pointed metric space. We define the Lipschitz homotopy group $\pi_{n}^{\mathrm{Lip}}\left(Y, y_{0}\right)$ in the same way as the classical homotopy group [20], with the exception that both mappings and homotopies are required to be Lipschitz. We do not require that the Lipschitz constant of the homotopy between Lipschitz mappings $f, g:\left(Q^{n}, \partial Q^{n}\right) \rightarrow\left(Y, y_{0}\right)$, where $Q^{n}=[0,1]^{n}$, is comparable to the larger of the Lipschitz constant of the mappings $f, g$.

$\pi_{0}^{\text {Lip }}\left(Y, y_{0}\right)$ has no group structure, it is the set of Lipschitz-path-connected components, i.e. components in which any two points can be connected by a rectifiable curve, and $\pi_{0}^{\mathrm{Lip}}\left(Y, y_{0}\right)=0$ means that the space is rectifiably connected. The following result is easy to prove [6].

Proposition 1.8. $\pi_{n}^{\mathrm{Lip}}\left(Y, y_{0}\right)=0$ if and only if every Lipschitz map $\left(\mathbf{S}^{n}, s_{0}\right) \rightarrow$ $\left(Y, y_{0}\right)$ admits a Lipschitz extension $\mathbf{B}^{n+1} \rightarrow Y$.

Here and in what follows $\mathbf{S}^{n}$ stands for the unit sphere $\mathbf{S}^{n}(0,1)$ in $\mathbf{R}^{n+1}$.

In the case of compact smooth manifolds Lipschitz homotopy groups are equivalent to the classical homotopy groups, because continuous mappings and homotopies can be approximated by smooth ones, however, for non-smooth spaces the situation is different. In [4] it was proved that $\pi_{n}^{\operatorname{Lip}}\left(\mathbf{H}_{n}\right) \neq 0$ and in [6] this fact was used to prove the corresponding lack of density of Lipschitz mappings: If $\mathcal{M}$ is a compact Riemannian manifold of dimension $\operatorname{dim} \mathcal{M} \geq n+1$, then Lipschitz mappings $\operatorname{Lip}\left(\mathcal{M}, \mathbf{H}_{n}\right)$ are not dense in $W^{1, p}\left(\mathcal{M}, \mathbf{H}_{n}\right)$ when $n \leq p<n+1$. Similarly using a generalized Hopf invariant it was proved in [16] that $\pi_{4 n-1}^{\mathrm{Lip}}\left(\mathbf{H}_{2 n}\right) \neq 0$ and the following result was concluded from it: If $\mathcal{M}$ is a compact Riemannian manifold of dimension $\operatorname{dim} \mathcal{M} \geq 4 n$, then Lipschitz mappings $\operatorname{Lip}\left(\mathcal{M}, \mathbf{H}_{2 n}\right)$ are not dense in $W^{1, p}\left(\mathcal{M}, \mathbf{H}_{2 n}\right)$ when $4 n-1 \leq p<4 n$. Thus the two non-density results are counterparts of Proposition 1.1 for the Heisenberg group targets, so one might expect that this result extends to other metric targets. Thus one might ask the following question for the class of mappings from a manifold $\mathcal{M}$ to a metric space $Y$ :

Question 1.9. Suppose that $\pi_{[p]}^{\operatorname{Lip}}(Y) \neq 0$ and $1 \leq p<\operatorname{dim} \mathcal{M}$. Is it true that Lipschitz mappings $\operatorname{Lip}(\mathcal{M}, Y)$ are not dense in $N^{1, p}(\mathcal{M}, Y)$ ?

We do not know the answer. One may ask also whether Proposition 1.3 and Theorem 1.6 extend to more general targets.

Question 1.10. Suppose that $\pi_{1}^{\mathrm{Lip}}(Y)=\ldots=\pi_{[p]}^{\mathrm{Lip}}(Y)=0$. Is it true that Lipschitz mappings $\operatorname{Lip}(\mathcal{M}, Y)$ are dense in $W^{1, p}(\mathcal{M}, Y)$ ?

It turns out that the answer is in the negative as the following results shows.

Theorem 1.11. For $n \geq 2$ there is a compact subset $Y \subset \mathbf{R}^{n+1}$ and a point $p \in Y \cap \mathbf{S}^{n}$ such that 
- $Y$ is homeomorphic to $\mathbf{S}^{n}$;

- $Y \backslash\{p\}$ is a smooth manifold diffeomorphic to $\mathbf{S}^{n} \backslash\{p\}$. In fact there is a Lipschitz continuous homeomorphism $\Phi: Y \rightarrow \mathbf{S}^{n}$ such that $\Phi: Y \backslash\{p\} \rightarrow$ $\mathbf{S}^{n} \backslash\{p\}$ is a smooth diffeomorphism;

- $\pi_{k}^{\mathrm{Lip}}(Y)=0$ for all $k \geq 1$ (for any choice of $y_{0}$ );

- Lipschitz mappings $\operatorname{Lip}\left(\mathbf{S}^{n}, Y\right)$ are not dense in $W^{1, n}\left(\mathbf{S}^{n}, Y\right)$.

In this result we consider the embedding of $Y$ into $\mathbf{R}^{n+1}$, but Corollary 2.7 in [10] shows also the lack of density if we would consider the Kuratowski embedding into $\ell^{\infty}$. This embedding is in a sense a canonical choice if we work with a general class of metric spaces.

Although $Y$ is Lipschitz homeomorphic to $\mathbf{S}^{n}$, it is not bi-Lipschitz homeomorphic to the sphere. The space $Y$ is not rectifiably connected: any curve in $Y$ connecting $p$ to another point has infinite length. In fact $Y$ has two Lipschitz-path-connected components. By adding a segment to $Y$ connecting $p$ with the antipodal point of $\mathbf{S}^{n}$, we obtain a set $Z$ that is rectifiably connected and has similar properties as $Y$.

Theorem 1.12. For $n \geq 2$ there is a compact set $Z \subset \mathbf{R}^{n+1}$ such that $\pi_{k}^{\text {Lip }}(Z)=$ 0 for all $k \geq 0$ and Lipschitz mappings $\operatorname{Lip}\left(\mathbf{S}^{n}, Z\right)$ are not dense in $W^{1, n}\left(\mathbf{S}^{n}, Z\right)$.

Thus in order to obtain positive density results we need a stronger condition than vanishing of Lipschitz homotopy groups. The next definition is taken from [31].

Definition 1.13. A metric space $Y$ is Lipschitz n-connected for some integer $n \geq 0$ if there is a constant $\gamma \geq 1$ such that for each $k \in\{0,1, \ldots, n\}$, every $L$ Lipschitz map $f: \mathbf{S}^{k} \rightarrow Y$ admits a $\gamma L$-Lipschitz extension $F: \mathbf{B}^{k+1} \rightarrow Y$.

The condition that the space is Lipschitz $n$-connected is stronger than vanishing of the Lipschitz homotopy groups for $k \leq n$, because we want to control the Lipschitz constant of the extension. As it will be explained later the set $Z$ is not Lipschitz $(n-1)$-connected despite the fact that all its Lipschitz homotopy groups are trivial.

Definition 1.14. The Nagata dimension $\operatorname{dim}_{N} X$ of a metric space $X$ is the least integer $n$ with the property that there is $C>0$ such that for any $s>0$, there is a covering $X=\bigcup_{i \in I} A_{i}$ such that

- $\operatorname{diam} A_{i} \leq C s$ for all $i \in I$;

- Every ball $B(x, s)$ intersects at most $n+1$ sets $A_{i}$.

If no such integer $n$ exists, then $\operatorname{dim}_{N} X=+\infty$.

The following result is easy to prove (see e.g. [31, Lemma 2.3])

Proposition 1.15. If $X$ is equipped with a doubling measure, then $\operatorname{dim}_{N} X<$ $\infty$

For spaces that are Lipschitz $n$-connected we have the following density result which in some sense is a counterpart of Proposition 1.3 and Theorem 1.6 but with an important additional restriction on the dimension of the domain, see also Question 1.10 .

Theorem 1.16. Suppose that the space $(X, d, \mu)$ supports the p-Poincare inequality and $\operatorname{dim}_{N} X \leq n$. If a separable metric space $Y$ is $\operatorname{Lipschitz}(n-1)$ connected, then Lipschitz mappings Lip $(X, Y)$ are dense in $N^{1, p}(X, Y)$ independently of the embedding of $Y$ into a Banach space. 
Remark 1.17. If a space supports the $p$-Poincaré inequality, then it supports the $q$-Poincaré inequality for all $p \leq q<\infty$ (by Hölder's inequality) and hence we have density in $N^{1, q}$ for all $q \geq p$. If in addition $X$ is complete and $p>1$, then there is $\varepsilon>0$ such that the space supports the $q$-Poincaré inequality for all $p-\varepsilon<q<\infty$, [28], and thus the density is true for this range of $q$.

Remark 1.18. If $X$ supports 1-Poincaré inequality, then we have density for all $1 \leq p<\infty$. This is consistent with results for mappings between manifolds: if $\pi_{1}(\mathcal{N})=\ldots=\pi_{n-1}(\mathcal{N})=0$, and $\operatorname{dim} \mathcal{M} \leq n$, then Proposition 1.3 gives density for $1 \leq p<n$, but if $p \geq n$ we always have density by the result of Schoen-Uhlenbeck $[33,34]$.

Remark 1.19. Theorems 1.16 and 1.12 show that the set $Z$ is not Lipschitz $(n-1)$-connected, but it can be checked more directly. Every Lipschitz mapping $f: \mathbf{S}^{k} \rightarrow Z, k \geq 0$ admits a Lipschitz extension $F: \mathbf{B}^{k+1} \rightarrow Z$, but using the construction of $Z$ one can show that for $k=0$ and $k=n-1$, there is no constant $C \geq 1$ with the property that every $L$-Lipschitz mapping $f: \mathbf{S}^{k} \rightarrow Z$ admits a $C L$ Lipschitz extension $F: \mathbf{B}^{k+1} \rightarrow Z$. This is a good exercise for the reader. We do not provide details here, because this direct argument will play no role in the paper.

Remark 1.20. According to [21, Proposition 2.13] Lipschitz $n$-connected sets in $\mathbf{R}^{n+1}$ are Lipschitz retracts of $\mathbf{R}^{n+1}$. This also follows from a more difficult result of Lang and Schlichenmaier, Lemma 2.1 below. From this fact the density of Lipschitz mappings in $W^{1, p}$ for all $1 \leq p<\infty$ easily follows, see also [13, Theorem 1.3].

It was proved in [38] that the Heisenberg group $\mathbf{H}_{n}$ is Lipschitz $(n-1)$-connected and hence we obtain

Corollary 1.21. Suppose that the space $(X, d, \mu)$ supports the p-Poincare inequality and $\operatorname{dim}_{N} X \leq n$. Then Lipschitz mappings $\operatorname{Lip}\left(X, \mathbf{H}_{n}\right)$ are dense in $N^{1, p}\left(X, \mathbf{H}_{n}\right)$.

This result generalizes Theorem 1.2(b) from [6], where the case of $X$ being a compact manifold was considered.

Notation is pretty standard. By $C$ we will denote a general constant whose value may change in a single string of estimates. The paper is organized as follows. In Section 2 we prove Theorem 1.16 and in Section 3 we prove Theorems 1.11 and 1.12.

\section{Proof of Theorem 1.16}

The following result due to Lang and Schlichenmaier [31, Theorem 1.5] will play a fundamental role in the proof.

Lemma 2.1. Suppose that $X$ and $Y$ are metric spaces such that $\operatorname{dim}_{N} X \leq n$ and $Y$ is Lipschitz $(n-1)$-connected. Then there is a constant $C \geq 1$ such that for any closed set $Z \subset X$ and any L-Lipschitz map $f: Z \rightarrow Y$ there is a CL-Lipschitz extension $F: X \rightarrow Y$.

Since the metric in $N^{1, p}(X, Y)$ is defined via an isometric embedding of $Y$ into a Banach space, we may assume that $Y \subset V$ is a subset of a Banach space $V$. Let $u \in N^{1, p}(X, Y) \subset N^{1, p}(X, V)$. Let $0 \leq g \in L^{p}(X)$ be an upper gradient of $u$. According to Proposition 1.4

$$
\|u(x)-u(y)\|_{V} \leq C d(x, y)\left(\left(\mathbf{M} g^{p}(x)\right)^{1 / p}+\left(\mathbf{M} g^{p}(y)\right)^{1 / p}\right) \quad \text { a.e. }
$$


Let

$$
E_{t}=\left\{x \in X:\left(\mathbf{M} g^{p}(x)\right)^{1 / p} \leq t\right\} .
$$

It follows from the weak type estimates of the maximal function that $t^{p} \mu\left(X \backslash E_{t}\right) \rightarrow 0$ as $t \rightarrow \infty$. Hence we can find a closed set $F_{t} \subset E_{t}$ such that $t^{p} \mu\left(X \backslash F_{t}\right) \rightarrow 0$. The function $u$ restricted to $F_{t}$ is $2 C t$-Lipschitz continuous. According to Lemma 2.1 there is a $C^{\prime} t$-Lipschitz extension $u_{t}: X \rightarrow Y$. It remains to prove that $\left\|u-u_{t}\right\|_{N^{1, p}} \rightarrow 0$ as $t \rightarrow \infty$. Since $X$ is bounded and the mapping $u_{t}$ is $C^{\prime} t$-Lipschitz, we conclude that $\left\|u_{t}\right\|_{V} \leq C+C t \operatorname{diam} X \leq C^{\prime}(1+t)$. Thus

$$
\int_{X}\left\|u-u_{t}\right\|_{V}^{p}=\int_{X \backslash F_{t}}\left\|u-u_{t}\right\|_{V}^{p} \leq C \int_{X \backslash F_{t}}\|u\|_{V}^{p}+C(1+t)^{p} \mu\left(\left\{X \backslash F_{t}\right\}\right) \rightarrow 0
$$

as $t \rightarrow \infty$. The following elementary lemma shows a localization property of upper gradients; the proof is quite standard and left to the reader.

Lemma 2.2. If $0 \leq g \in L^{p}(X)$ is an upper gradient of $f \in N^{1, p}(X, V)$ and $f$ is constant on a closed set $E \subset X$, then $h=g \chi_{X \backslash E}$ is an upper gradient of $f$.

Since $u_{t}$ is $C t$-Lipschitz, the constant function $C t$ is its upper gradient and since $u-u_{t}=0$ on the closed set $F_{t}$ it follows from the lemma that $h=(g+C t) \chi_{X \backslash F_{t}}$ is an upper gradient of $u-u_{t}$. We have

$$
\int_{X} h^{p} d \mu \leq \int_{X \backslash F_{t}}(g+C t)^{p} d \mu \rightarrow 0
$$

as $t \rightarrow \infty$. Thus $\left\|u-u_{t}\right\|_{N^{1, p}} \rightarrow 0$ as $t \rightarrow \infty$. The proof is complete.

\section{Example}

In this section we will prove Theorems 1.11 and 1.12. In the first subsection we will provide details of the construction of the set $Y$ and we will prove all its properties except the lack of density of Lipschitz mappings. In the second subsection we will construct a set $Z$ and we will prove that $\pi_{k}^{\operatorname{Lip}}(Z)=0$ for all $k \geq 0$. In the last subsection we will prove the lack of density of Lipschitz mappings in $W^{1, n}\left(\mathbf{S}^{n}, Y\right)$ and $W^{1, n}\left(\mathbf{S}^{n}, Z\right)$.

3.1. Construction of $\boldsymbol{Y}$. The main idea is to pick a point $p \in \mathbf{S}^{n}$ and add continuous oscillations into the normal direction around that point. The oscillations should be so that the resulting set is still homeomorphic to the sphere with the homeomorphism $\phi$ being in $W^{1, n}$.

The amplitude of the oscillations is described by $a \in C^{0}([0, \infty)) \cap C^{\infty}((0, \infty))$

$$
a(t):= \begin{cases}0, & t=0, \\ \frac{\sin (\log (\log (e / t)))}{1+\log (\log (e / t))}, & t \in(0,1], \\ \text { smooth extension, } & t \in(1,4 / 3), \\ 0, & t \geq 4 / 3 .\end{cases}
$$

Fix a point $p \in \mathbf{S}^{n}$ and define $\phi: \mathbf{S}^{n} \rightarrow \mathbf{R}^{n+1}$ by

$$
\phi(x)=\left(1+\frac{1}{2} a(|x-p|)\right) x .
$$


The set $Y$ is defined as the image of the mapping $\phi, Y=\phi\left(\mathbf{S}^{n}\right)$. Since $\phi \in$ $C^{0}\left(\mathbf{S}^{n}, \mathbf{R}^{n+1}\right)$ translates points of the sphere along the direction of the outer normal to the sphere, $\phi$ has no self-intersections and thus it is a homeomorphism. Clearly $Y \backslash\{p\}$ is a smooth manifold. Let $\Phi=\phi^{-1}: Y \rightarrow \mathbf{S}^{n}$ be the inverse homeomorphism. Obviously, $\Phi$ is the restriction of the projection mapping

$$
\pi(x)=\frac{x}{|x|} \in C^{\infty}\left(\mathbf{R}^{n+1} \backslash\{0\}, \mathbf{S}^{n}\right)
$$

to $Y$. Hence $\Phi$ is Lipschitz continuous and its restriction to $Y \backslash\{p\}$ is a smooth diffeomorphism. The function $a$ was chosen in order to satisfy the claim of the next lemma.

\section{Lemma 3.1.}

$$
\int_{0}^{s}\left|a^{\prime}(t)\right| d t=\infty \quad \text { for any } s>0
$$

but

$$
\int_{0}^{1}\left|a^{\prime}(t)\right|^{n} t^{n-1} d t<\infty \quad \text { for all } n \geq 2
$$

Proof. Since

$$
(\log (\log (e / t)))^{\prime}=-\frac{1}{t \log (e / t)}
$$

for $0<t<1$ we have

$$
a^{\prime}(t)=\frac{-\cos (\log (\log (e / t)))}{t \log (e / t) \cdot(1+\log (\log (e / t)))}+\frac{\sin (\log (\log (e / t)))}{t \log (e / t) \cdot(1+\log (\log (e / t)))^{2}} .
$$

Thus for any $0<s<1$ the change of variables $x(t)=\log (\log (e / t))$ yields

$$
\int_{0}^{s}\left|a^{\prime}(t)\right| d t=\int_{x(s)}^{\infty}\left|\frac{-\cos x}{x+1}+\frac{\sin x}{(x+1)^{2}}\right| d x \geq \int_{x(s)}^{\infty} \frac{|\cos x|}{x+1} d x-\int_{x(s)}^{\infty} \frac{d x}{(x+1)^{2}}=\infty .
$$

On the other hand for $n \geq 2$ we have

$$
\int_{0}^{1}\left|a^{\prime}(t)\right|^{n} t^{n-1} d t \leq 2^{n} \int_{0}^{1} \frac{d t}{t \log ^{n}(e / t)}=2^{n} \int_{1}^{\infty} \frac{d x}{x^{n}}<\infty .
$$

The proof is complete.

Lemma 3.2. $\phi \in W^{1, n}\left(\mathbf{S}^{n}, Y\right)$.

Proof. It suffices to show that the derivative of $\phi$ is integrable with the exponent $n$ in some neighborhood of the singularity $p$. Using a suitable coordinate system in a neighborhood of $p \in \mathbf{S}^{n}$ both in the domain and in the target $\mathbf{R}^{n+1}$, the image of $\phi$ becomes the graph of the function $a(|x|)$. The fact that the function $a(|x|)$ has derivative integrable with exponent $n$ follows from (3.3) after integration in the polar coordinate system:

$$
\int_{\mathbf{B}^{n}(0,1)}|\nabla(a(|x|))|^{n} d x=C \int_{0}^{1}\left|a^{\prime}(t)\right|^{n} t^{n-1} d t<\infty .
$$

The proof is complete.

In the last subsection we will prove that the mapping $\phi \in W^{1, n}\left(\mathbf{S}^{n}, Y\right)$ cannot be approximated by Lipschitz maps $\operatorname{Lip}\left(\mathbf{S}^{n}, Y\right)$ in the Sobolev norm. But now we 
will prove that $\pi_{k}^{\mathrm{Lip}}(Y)=0$ for all $k \geq 1$. This immediately follows from the next lemma.

Lemma 3.3. If $k \geq 1$ and $f: \mathbf{S}^{k} \rightarrow Y$ is Lipschitz continuous, then $f\left(\mathbf{S}^{k}\right)=\{p\}$ or $p \notin f\left(\mathbf{S}^{k}\right)$.

Indeed, if $p \notin f\left(\mathbf{S}^{k}\right)$, then the image of $\phi$ omits a certain neighborhood of $p$ and hence it is contained in a subset of $Y$ which is diffeomorphic to $\mathbf{B}^{n}$ and thus Lipschitz contractible.

Proof of Lemma 3.3. Since $f$ is Lipschitz, any two points in the image of $f\left(\mathbf{S}^{k}\right)$ can be connected by a rectifiable curve. Thus it suffices to show that the point $p \in Y$ cannot be connected to any other point in $q \in Y$ by a rectifiable curve. Using the coordinate system near $p$ as in the previous proof we can represent a neighborhood of $p \in Y$ as the graph of the function $a(|x|)$ defined in $\mathbf{B}^{n}$. In this coordinate system $p=0$. Consider the plane through $p$ and $q$ that is orthogonal to $\mathbf{B}^{n}$. The curve obtained in the intersection of this plane with the graph of $a(|x|)$ is the graph of the function $a(t)$ of one variable. This curve connects $p$ to $q$. If the Euclidean distance of the projections of $p$ and $q$ onto $\mathbf{B}^{n}$ equals $s$, the length of this curve is

$$
\int_{0}^{s} \sqrt{1+a^{\prime}(t)^{2}} d t=\infty
$$

by (3.2). Note that no other curve connecting $p$ to $q$ can be shorter. The proof of the lemma is complete. This also completes the proof of the fact that $\pi_{k}^{\text {Lip }}(Y)=0$ for $k \geq 1$.

3.2. Construction of $Z$. In this section we will provide the construction of the set $Z$ from Theorem 1.12 and we will prove that $\pi_{k}^{\text {Lip }}(Z)=0$ for all $k \geq 0$. The proof of the lack of density will be given in the next section. The set $Z$ is simply obtained from $Y$ by adding the segment $\overline{p q}$ that connects the point $p$ through the center of the sphere with the antipodal point $q$ of $\mathbf{S}^{n}$. Note that this antipodal point belongs also to $Y$. Clearly the set $Z$ is now rectifiably connected since the point $p$ can be connected with the rest of the set through the added segment, so $\pi_{0}^{\operatorname{Lip}}(Z)=0$. The fact that $\pi_{k}^{\operatorname{Lip}}(Z)=0$ for $k \geq 1$ is also easy. If $f: \mathbf{S}^{k} \rightarrow Z$ is Lipschitz, then $f\left(\mathbf{S}^{k}\right)$ is contained in $Y$ with a neighborhood of $p$ removed, plus $\overline{p q}$, see Lemma 3.3. This set is Lipschitz contractible.

3.3. Proof of the lack of density of Lipschitz maps. In this section we will complete the proofs of Theorems 1.11 and 1.12 by showing the lack of density of Lipschitz mappings.

Proof of Theorem 1.11. We will prove that the mapping $\phi \in W^{1, n}\left(\mathbf{S}^{n}, Y\right)$ defined in (3.1) cannot be approximated by Lipschitz mappings $\operatorname{Lip}\left(\mathbf{S}^{n}, Y\right)$ in the $W^{1, n}$ norm. Suppose to the contrary that there is a sequence $g_{k} \in \operatorname{Lip}\left(\mathbf{S}^{n}, Y\right)$ such that

$$
\left\|\phi-g_{k}\right\|_{1, n} \rightarrow 0 \quad \text { as } k \rightarrow \infty .
$$

By Lemma 3.3, $p \notin g_{k}\left(\mathbf{S}^{n}\right)$. As we know $\pi=\Phi=\phi^{-1}: Y \rightarrow \mathbf{S}^{n}$ is smooth in a neighborhood of $Y$ in $\mathbf{R}^{n+1}$. Hence $f_{k}:=\Phi \circ g_{k} \in \operatorname{Lip}\left(\mathbf{S}^{n}, \mathbf{S}^{n} \backslash\{p\}\right), \Phi \circ \phi=$ id: $\mathbf{S}^{n} \rightarrow \mathbf{S}^{n}$ and (3.4) yields

$$
\left\|f_{k}-\mathrm{id}\right\|_{1, n} \rightarrow 0 \quad \text { as } k \rightarrow \infty .
$$


The degree of a Lipschitz mapping can be expressed as the integral of the Jacobian. The integral of the Jacobian is continuous in the $W^{1, n}$ norm. That easily follows from the definition of the Jacobian and Hölder's inequality. Hence (3.5) implies that the degree of the mapping $f_{k}$ converges to the degree of the identity mapping, i.e. it converges to 1 , but this is impossible, because the mapping $f_{k}$ is not surjective and hence its degree equals zero. The proof is complete.

Proof of Theorem 1.12. Now we will prove that Lipschitz mappings $\operatorname{Lip}\left(\mathbf{S}^{n}, Z\right)$ are not dense in $W^{1, n}\left(\mathbf{S}^{n}, Z\right)$. Namely we will prove that $\phi \in W^{1, n}\left(\mathbf{S}^{n}, Y\right) \subset$ $W^{1, n}\left(\mathbf{S}^{n}, Z\right)$ cannot be approximated by Lipschitz mappings $\operatorname{Lip}\left(\mathbf{S}^{n}, Z\right)$. To the contrary suppose that $g_{k} \in \operatorname{Lip}\left(\mathbf{S}^{n}, Z\right)$ is a Lipschitz approximation. The image of $g_{k}$ is contained in $Y$ with a neighborhood of $p$ removed, plus $\overline{p q}$. Thus composing $g_{k}$ with the retraction of $\overline{p q}$ onto $q$ gives a Lipschitz map $\tilde{g}_{k}: \mathbf{S}^{n} \rightarrow Y$. Since $g_{k} \rightarrow \phi$ in $L^{n}$, it easily follows that the measure of the set $g_{k}^{-1}(\overline{p q})$ converges to zero as $k \rightarrow \infty$. Since $\tilde{g}_{k}$ differs from $g_{k}$ only on the set $g_{k}^{-1}(\overline{p q})$ one can easily show that $\left\|g_{k}-\tilde{g}_{k}\right\|_{1, n} \rightarrow 0$ and hence $\left\|\tilde{g}_{k}-\phi\right\|_{1, n} \rightarrow 0$. Now the result follows directly from the previous proof, because Lipschitz mappings $\tilde{g}_{k}$ into $Y$ cannot approximate $\phi$ in the $W^{1, n}$ norm.

\section{References}

[1] Ambrosio, L.: Metric space valued functions of bounded variation. - Ann. Sc. Norm. Super. Pisa Cl. Sci. 17, 1990, 439-478.

[2] Bethuel, F.: The approximation problem for Sobolev maps between two manifolds. - Acta Math. 167, 1991, 153-206.

[3] Bethuel, F., and X. M. Zheng: Density of smooth functions between two manifolds in Sobolev spaces. - J. Funct. Anal. 80, 1988, 60-75.

[4] Balogh, Z., and K. S. Fässler: Rectifiability and Lipschitz extensions into the Heisenberg group. - Math. Z. 263, 2009, 673-683.

[5] Capogna, L., and F.-H. Lin: Legendrian energy minimizers. I. Heisenberg group target. Calc. Var. Partial Differential Equations 12, 2001, 145-171.

[6] DeJarnette, N., P. HajŁasz, L. Lukyanenko, and J. T. Tyson: On the lack of density of Lipschitz mappings in Sobolev spaces with Heisenberg target. - arXiv:1109.4641.

[7] Eells, J., and B. Fuglede: Harmonic maps between Riemannian polyhedra. - Cambridge Tracts in Math. 142, Cambridge Univ. Press, Cambridge, 2001.

[8] Eells, J., and L. Lemaire: A report on harmonic maps. - Bull. London Math. Soc. 10, 1978, $1-68$.

[9] Gromov, M., and R. Schoen: Harmonic maps into singular spaces and $p$-adic superrigidity for lattices in groups of rank one. - Inst. Hautes Études Sci. Publ. Math. 76, 1992, 165-246.

[10] HajŁasz, P.: Sobolev mappings: Lipschitz density is not an isometric invariant of the target. - Int. Math. Res. Not. IMRN 2011:12, 2011, 2794-2809.

[11] HajŁasz, P.: Sobolev mappings between manifolds and metric spaces. - In: Sobolev spaces in mathematics. I, Int. Math. Ser. (N. Y.) 8, Springer, New York, 2009, 185-222.

[12] HajŁasz, P.: Density of Lipschitz mappings in the class of Sobolev mappings between metric spaces. - Math. Ann. 343, 2009, 801-823.

[13] HajŁasz, P.: Sobolev mappings: Lipschitz density is not a bi-Lipschitz invariant of the target. - Geom. Funct. Anal. 17, 2007, 435-467.

[14] HajŁasz, P.: Approximation of Sobolev mappings. - Nonlinear Anal. 22, 1994, 1579-1591.

[15] HajŁasz, P., and P. Koskela: Sobolev met Poincaré. - Mem. Amer. Math. Soc. 688, 2000, $1-101$. 
[16] HajŁasz, P., A. Schikorra, and J. T. Tyson: Homotopy groups of spheres and Lipschitz homotopy groups of Heisenberg groups. - Geom. Funct. Anal. 24, 2014, 245-268.

[17] HajŁasz, P., and J. T. Tyson: Sobolev Peano cubes. - Michigan Math. J. 56, 2008, 687-702.

[18] Hang, F., and F. Lin: Topology of Sobolev mappings. - Math. Res. Lett. 8, 2001, 321-330.

[19] Hang, F., and F. Lin: Topology of Sobolev mappings II. - Acta Math. 191, 2003, 55-107.

[20] Hatcher, A.: Algebraic topology. - Cambridge Univ. Press, Cambridge, 2002.

[21] Heinonen, J.: Lectures on Lipschitz analysis. - Report. University of Jyväskylä Department of Mathematics and Statistics, 100. Univ. of Jyväskylä, Jyväskylä, 2005.

[22] Heinonen, J., and P. Koskela: Quasiconformal maps in metric spaces with controlled geometry. - Acta Math. 181, 1998, 1-61.

[23] Heinonen, J., P. Koskela, N. Shanmugalingam, and J. T. Tyson: Sobolev classes of Banach space-valued functions and quasiconformal mappings. - J. Anal. Math. 85, 2001, 87139.

[24] Jost, J.: Equilibrium maps between metric spaces. - Calc. Var. 2, 1994, 173-205.

[25] Jost, J.: Generalized Dirichlet forms and harmonic maps. - Calc. Var. 5, 1997, 1-19.

[26] Jost, J.: Nonlinear Dirichlet forms. - In: New directions in Dirichlet forms, AMS/IP Stud. Adv. Math. 8, Amer. Math. Soc., Providence, RI, 1998, 1-47.

[27] Jost, J., and K. Zuo: Harmonic maps into Bruhat-Tits buildings and factorizations of $p$ adically unbounded representations of $\pi_{1}$ of algebraic varieties. I. - J. Algebraic Geom. 9, 2000, $1-42$.

[28] Keith, S., and X. Zhong: The Poincaré inequality is an open ended condition. - Ann. of Math. (2) 167, 2008, 575-599.

[29] KorevaAr, N. J., and R. M. Schoen: Sobolev spaces and harmonic maps for metric space targets. - Comm. Anal. Geom. 1, 1993, 561-659.

[30] Kronz, M.: Some function spaces on spaces of homogeneous type. - Manuscripta Math. 106, 2001, 219-248.

[31] Lang, U., and T. Schlichenmaier: Nagata dimension, quasisymmetric embeddings, and Lipschitz extensions. - Int. Math. Res. Not. IMRN 2005:58, 2005, 3625-3655.

[32] Reshetnyak, Yu. G.: Sobolev classes of functions with values in a metric space. - Sibirsk. Mat. Zh. 38, 1997, 657-675 (in Russian), English translation in Siberian Math. J. 38, 1997, $567-583$.

[33] Schoen, R., and K. Uhlenbeck: The Dirichlet problem for harmonic maps. - J. Diff. Geom. 18, 1983, 153-268.

[34] Schoen, R., and K. Uhlenbeck: Approximation theorems for Sobolev mappings. - Unpublished manuscript.

[35] Serbinowski, T.: Boundary regularity of harmonic maps to nonpositively curved metric spaces. - Comm. Anal. Geom. 2, 1994, 139-153.

[36] Shanmugalingam, N.: Newtonian spaces: an extension of Sobolev spaces to metric measure spaces. - Rev. Mat. Iberoam. 16, 2000, 243-279.

[37] Troyanov, M.: Approximately Lipschitz mappings and Sobolev mappings between metric spaces. - In: Proceedings on Analysis and Geometry (Novosibirsk Akademgorodok, 1999), Izdat. Ross. Akad. Nauk Sib. Otd. Inst. Mat., Novosibirsk, 2000, 585-594 (in Russian).

[38] Wenger, S., and R. Young: Lipschitz extensions into jet space Carnot groups. - Math. Res. Lett. 17, 2010, 1137-1149.

Received 25 June 2013 • Accepted 17 December 2013 